REVISTA DE LA UNIVERSIDAD DEL AZUAY

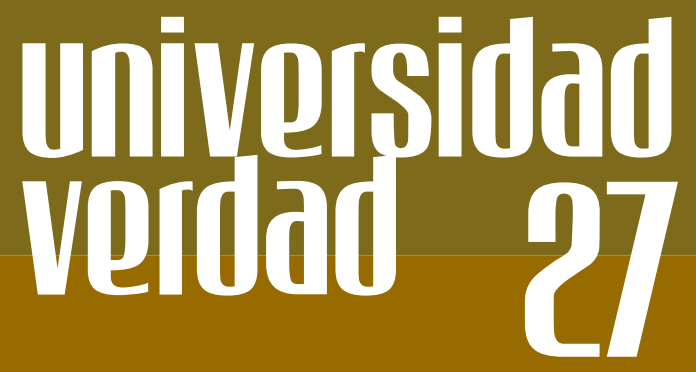

UNIVERSIDAD

DEL AZUAY

\title{
CULTURA, PATRIMONIO, IDENTIDAD Y DESARROLLO
}

\author{
Janeth Molina $C$.
}

Candidata a Master en Estudios de la Cultura 


\section{Formulación del problema:}

El ámbito de la cultura es un tema de difícil análisis por los cambios y transformaciones que ocurren y modifican su sentido, sus campos y sus objetos. Las discusiones conceptuales se replantean en una modernidad tardía en la que las fronteras de los estadosnación son cada vez más difusas, las temporalidades culturales diversas, y las certezas sobre lo propio y ajeno en el nivel cultural se han vuelto borrosas.

Más aún, el debate se inserta en un contexto de mundialización, definida como: "la intensificación de las relaciones sociales en todo el mundo por las que se enlazan lugares lejanos, de tal manera que los acontecimientos locales están configurados por acontecimientos que ocurren a muchos kilómetros de distancia o viceversa". ${ }^{1}$

Vivimos en un contexto de cambios vertiginosos en el que se amalgaman o entran en contradicción lo global con lo local, en el que si por un lado se producen procesos de homogeneización cultural, por otro, y como respuesta contestataria, planificada o no, se da el resurgimiento y la afirmación de heterogeneidades que insisten en recuperar su memoria y tradiciones culturales propias.

El sistema-mundo es percibido por nosotros como un proceso sinuoso, que se moviliza rápidamente y cambia de perfiles constantemente. Sin embargo a pesar de estos procesos que inciden directamente en las formas de vida cotidiana de la gente, las instituciones de mediación cultural más importantes de Ecuador y de la ciudad, por diversos motivos, parecería que no logran sintonizar sus propuestas con los cambios que están ocurriendo en la cultura, en las concepciones, en las percepciones y en los hábitos de la gente, viviendo de "glorias del pasado". 
En Cuenca, por ejemplo, los estudios antropológicos, los de crítica literaria o pictórica, las discusiones sobre temas y propuestas culturales, han disminuido considerablemente durante estos años. A pesar de que con la Declaratoria, la ciudad se debería perfilar y posicionar de diferente manera, en el contexto anotado, hasta el momento, no se han definido políticas culturales ni un plan de desarrollo local para la cultura que esté engarzado en un plan de desarrollo global de Cuenca. No existen propuestas organizacionales diferentes y las instituciones culturales siguen estableciendo sus agendas de trabajo aisladas entre sí y de los diversos actores.

Temas tales como globalización, descentralización y cultura, en el marco de la Declaratoria podrían generar inmensas posibilidades en el marco de un proyecto alternativo de desarrollo, sin embargo no forman parte de las agendas de debate.

Es evidente que la mediación cultural -entendida como la capacidad de facilitar procesos de producción y apropiación de la cultura material y simbólica- no pasa solo por lo que instituciones tales como la Casa de la Cultura, las universidades, las organizaciones gubernamentales y no gubernamentales realizan, sino, y quizá en mayor medida, por la casa, la escuela, el barrio, la comunidad, los medios de comunicación que van conformando los "habitus" de los ciudadanos cuencanos.

Sin embargo, en ciudades como la nuestra, lo que propongan y activen estas instituciones incidirá en mayor o en menor medida en las prácticas culturales, por ello la importancia de acercarnos a una reflexión sobre las concepciones que subyacen en las prácticas de instituciones culturales cuencanas; más concretamente, intentaré analizar las concepciones sobre cultura, identidad, patrimonio y desarrollo que subyacen en las prácticas de las principales instituciones culturales en Cuenca, tomando como referencia los tres últimos años en los que se plantea y se consigue que Cuenca sea declarada como Patrimonio Cultural de la Humanidad.

Ahora bien, la discusión conceptual puede generar discusiones 
"bizantinas" porque como lo señala Ticio Escobar hay que enfrentar "ciertos conceptos que, aún confusos y molestos, integran el paisaje semántico de donde inevitablemente partimos y expresan las contradicciones y ambigüedades que anegan vastas regiones de nuestra incipiente teoría", algunas categorías son problemáticas y discutibles pero no pueden ser soslayadas. ${ }^{2}$

Pero por difícil que se presente la discusión, esta es previa, pues clarificar el uso de términos y conceptos, conocer los criterios desde los que se vinculan temas tales como identidad, patrimonio y desarrollo local, pueden proporcionarnos pistas para aterrizar en el abordaje de políticas culturales y en la organización de un Plan de Desarrollo de la cultura local, propuestas que empiezan a perfilarse como indispensables en la ciudad. De lo contrario en el camino se presentarán malentendidos y desencuentros o, lo que es peor, en nombre de la tradición, del patrimonio y de la cultura cuencana se pueden generar prácticas culturales distorsionadoras ligadas básicamente a procesos mercantiles, profundas modificaciones en los significados, que vía mercado global conviertan al patrimonio cultural en un gran "simulacro": artesanías copiadas de otros territorios culturales, turismo cultural que contrata actores para que sean los "curanderos o shamanes", turismo ecológico depredatorio, etc.

Motivada por estas reflexiones me propongo realizar un primer acercamiento a conceptos que se encuentran en las ponencias y los proyectos publicados por el Programa Interinstitucional "La identidad en el Patrimonio", organizado por la Casa de la Cultura "Benjamín Carrión", Núcleo del Azuay, la Sucursal en Cuenca del Banco Central y la Universidad del Azuay (1999-2000) y además reforzar el análisis de los conceptos en base a entrevistas realizadas a directivos y autoridades de la ciudad.

El documento señalado recoge 3 ponencias sobre "Cultura, Identidad y Patrimonio" discutidas en los foros y 26 proyectos que las instituciones culturales cuencanas realizarían durante el año 2000, como una respuesta colectiva para superar los problemas de desarticulación interinstitucional y ante la Declaratoria de Cuenca 
como Patrimonio Cultural de la Humanidad, por parte de la UNESCO. Los proyectos se articularon en cinco grandes áreas: Arte y artesanía (6), Preservación de Patrimonio (6), Educación (7), Comunicación (2) y Línea Editorial (5).

\section{El Programa "La identidad en el Patrimonio"}

En una rápida evaluación de lo que ocurría en el campo cultural en Cuenca, en las reuniones del Comité Interinstitucional, en 1999, se señalaba entre otros los siguientes problemas:

La inexistencia de propuestas culturales a largo plazo, una oposición entre "arte culto" cuya difusión se privilegia y "arte o cultura popular" concebida como una expresión menos valiosa, informal y espontánea de la creatividad, una tendencia a circunscribir el patrimonio cultural a lo arquitectónico, difusión cultural dirigida hacia los públicos instruidos de la ciudad, hacia la elite, y desconocimiento de las expectativas de la comunidad protagonismos institucionales, presupuestos exiguos, activismo cultural sin horizonte conceptual que guía y potencie sus propuestas. Inexistencia de debate en torno a la cultura, falta de criticidad, lo que prova un discurso de elogios mutuos. La cultura autonomizada del debate político.

Buscando revertir esta situación y sobre todo para articular esfuerzos en torno a la Declaratoria, la Casa de la Cultura, "Benjamín Carrión", Núcleo del Azuay, el Banco Central del Ecuador, Sucursal Cuenca y la Universidad del Azuay se propusieron motivar cambios en esta realidad descrita muy someramente y convocaron a 30 instituciones culturales cuencanas para debatir en torno a temas de interés conjunto y motivar a que las instituciones, desde sus propias particularidades y especificidades, realicen propuestas para un plan de desarrollo para la cultura local.

La respuesta inicial fue interesante, alrededor de 30 instituciones (de las 38 que constan en el Directorio de instituciones y proyectos 
de desarrollo cultural realizado en 1997, por Habit-Com, con el auspicio de la UNESCO) ${ }^{3}$ participaron en los talleres y encuentros. EI resultado fue un primer proceso de discusión en torno a temas claves tales como identidad, cultura y patrimonio, que serviría de guía -en el plano conceptual- para la formulación de 26 proyectos.

En un primer acercamiento al documento se percibe que a nivel conceptual existe una diversidad de concepciones en torno a cultura, identidad, patrimonio y desarrollo, lo que ocasiona que los proyectos presentados por las diversas instituciones no logren vertebrarse como un plan sólido para la cultura local.

A un año de este proceso, insisto en que la construcción de proyectos no son suficientes, y por lo tanto el esfuerzo inicial de coordinación habría que reforzarlo con un debate constructivo y propositivo en torno a ejes centrales que fundamenten el trabajo interinstitucional, integrando a nivel conceptual análisis antropológicos y sociológicos como única forma de abordar la realidad de la cultura de una forma integral. Esto permitirá, vertebrar -desde el contexto en el que se desarrolla la cultura, esto es, un contexto en el que lo local y lo global se implican mutuamente- una propuesta a largo plazo.

\section{Objetivos:}

1. Conocer y analizar los conceptos que en torno a cultura, patrimonio, identidad y desarrollo tienen las principales instituciones culturales a nivel local.

2. Señalar las implicaciones que estos discursos generan en las prácticas.

3. Delinear una propuesta que articule conceptos y prácticas para el desarrollo de la cultura local. 


\section{LOS DISCURSOS Y LAS PRÁCTICAS}

\section{Sobre el concepto de cultura}

Señalar un concepto de cultura como el más importante no es posible por la polivalencia que el término tiene en la sociedad contemporánea y por las múltiples dimensiones que puede connotar.

En general se ha señalado que el término, ha significado primero "un estado general o habito de la mente, manteniendo relaciones próximas con la idea de perfección humana". En segundo lugar, vino a significar "el estado general de desarrollo intelectual en una sociedad como un todo". En tercer lugar vino a significar "el cuerpo general de las artes" (...) y en cuarto lugar, vino a significar "un modo de vida global, material, intelectual y espiritual". ${ }^{4}$

La discusión conceptual ha tenido un largo recorrido, matizada por diferentes visiones del mundo a nivel de diversas disciplinas sociales, científicas y políticas.

Parecería sin embargo que en nuestro país y más concretamente en la ciudad se han diferenciado claramente dos posiciones. Una concepción que concibe lo cultural como ligado al ámbito artes a la que se califica como concepción tradicional y otra más global, que se desarrolla desde la antropología cultural y que hace referencia a todas las producciones materiales y simbólicas de la humanidad.

El término cultura, en el documento analizado se enmarca en estas dos acepciones:

a) En el sentido antropológico: "conjunto de ideas, creencias, actitudes, valores jerarquizados, tecnologías y sistemas de pensamiento y comunicación, de acuerdo con los cuales organizamos nuestras vidas como parte de grupos estructurados". ${ }^{5}$

Esta concepción, por lo menos en el plano del discurso, no así en la práctica, es probablemente la concepción hegemónica entre las 
instituciones culturales de Cuenca. Así la más importante institución cultural en Cuenca, la asa de la Cultura Ecuatoriana, Benjamín Carrión, Núcleo del Azuay señala:

"El punto de partida es la cultura desde una visión antropológica, como el conjunto de manifestaciones, de expresiones de una comunidad, lo que implica tanto la presencia de lo que es la cultura material y la intangible". ${ }^{6}$

En el análisis que el Municipio de Cuenca, hace respecto del término cultura aplicado a la ciudad se puede apreciar también como se lo aborda:

"Yo empezaría poniéndole en plural a la palabra (cultura) porque me parece que ventajosamente hemos ido entendiendo que somos producto de la diversidad que tiene enorme impacto en la vida nacional y realmente las culturas que están predominando en el Ecuador no siempre han sido reconocidas. Unas han estado actuando y otras han estado inclusive siendo vulneradas y criticadas por una de estas formas culturales predominantes". ${ }^{7}$

La visión que antecede parte desde una visión antropológica, esto es de la valoración de lo otro, de lo diverso, reconociendo el etnocentrismo que ha existido, pero además nos remite a otros temas tales como el análisis de las estructuras de poder, y por lo tanto de la hegemonía lograda en torno al capital simbólico.

Este abordaje, si se lo realiza con profundidad y tomando como base los análisis de la sociología e la cultura, podría generar interesantes perspectivas de análisis de lo que sucede en la Cuenca de hoy y por lo tanto generar diferentes propuestas en las políticas culturales de Cuenca a más largo plazo.

A pesar del análisis anotado, el Municipio plantea la necesidad de unificar las prácticas culturales a través de un organismo que lo resolvería todo: 
"Eta cultura de la diversidad, esta forma de enfrentar el desarrollo cultural obliga también a no considerar individuos sino colectivos. Yo aspiro desde la municipalidad a impulsar un Consejo de Culturas en Cuenca, y ojalá puede hacer de esto una constante nacional". ${ }^{8}$

Lo anotado contradice la percepción que se tiene de los procesos culturales, pues sin investigaciones sobre las diversas culturas, sin comprensión global de la diversidad, sin políticas públicas definidas a nivel cultural, la propuesta de un mecanismo institucional unificador podría inclusive disolver las diferencias y la diversidad a la que se refiere esta institución en sus análisis.

b) Una segunda concepción presenta un cierto grado de ambigüedad pues no tiene inconveniente en plantear las dos acepciones, la tradicional y la antropológica, conjuntamente.

(...) "El Banco Central se maneja en una polisemia en el ámbito de la definición de cultura: una noción de cultura como bellas artes, promocionamos el arte como creación elitista... pero acogemos la definición de cultura en el sentido antropológico, como herencia social (...); esto está ligado a las actividades y a las reservas específicas que mantiene el Banco y que son lo que tiene que ver con el patrimonio cultural tangible, esto es la arqueología, arte, etnografía y numismática". 9

Los ejemplos anotados nos permiten señalar que las instituciones manejan diversas concepciones sobre cultura: una antropológica, que supera la noción de cultura asociada exclusivamente al arte, los buenos modales o la erudición y otra que, o bien se mantienen en la definición tradicional de cultura (las artes bellas) o bien mezcla dos concepciones diferentes como lo ejemplifica lo anteriormente señalado. Esto representa un primer problema pues evidencia insuficiencias conceptuales y demuestra que efectivamente algunas instituciones realizan sus propuestas sin un claro referente conceptual que guíe su accionar. 
De esto se deriva claramente que estas concepciones inciden directamente en las prácticas cotidianas. Así, al revisar los proyectos presentados en este plan y clasificarlos de acuerdo a su principal perfil encontramos que de los 25 proyectos apenas dos tienen que ver con la concepción tradicional de cultura referida a lo artístico: uno de video y otro que pretende producir 4 obras de arte integrales esto es el desarrollo en una misma obra de teatro, danza, música tradicional, video, performances, arte objetual, etc., intentando producir renovación en este nivel.

Esto constituye un interesante síntoma de un cambio en la concepción de cultura, que a mi modo de ver se ha producido durante los últimos años en Cuenca y nos permite concluir que desde una concepción de cultura ligada exclusivamente a las artes se ha pasado a otra, antropológica, que engloba diversas manifestaciones de la vida social. Habría sin embargo que ver cómo este giro conceptual ha repercutido en la vida cotidiana de la ciudad, en las propuestas institucionales en la oferta, recepción y el consumo de productos culturales, etc.

Las nociones de cultura que tienen las instituciones culturales las lleva también a especializarse en determinados ámbitos, por la imposibilidad de abarcar diversos aspectos y generar planteamientos globales. Así por ejemplo, la Casa de la Cultura "dentro de las manifestaciones de la cultura, ha asumido aquellas tareas que tienen que ver con el arte en sus más amplias manifestaciones", o el Banco Central, difunde todo lo que sea cultura en general en base de lo que es patrimonio tangible.

El análisis precedente evidencia que concepciones, políticas y prácticas culturales están ligadas entre sí y articulan el perfil institucional y las políticas culturales de las principales entidades a nivel cultural, pero también nos lleva a señalar la necesidad de profundizar en la conceptualización de la cultura desde una forma más integral y multidimensional e investigar la realidad local desde esta perspectiva. 
Esto podría generarse si a la visión antropológica, valorada por captar el carácter constitutivo y constituyente de la cultura, por develar el etnocentrismo y promover la dignidad de todas las culturas, pero criticada por su tendencia a unificar y armonizar las culturas a nivel interno y olvidar la perspectiva de poder y la dominación generando un relativismo cultural, le sumamos la perspectiva sociológica del análisis cultural lo que implicará un abordaje integral y multidimensional de lo que sucede realmente en nuestro medio, pues como argumenta Thompson: "las formas culturales no son solo pautas de significado sino que se hallan insertas en relaciones de poder y de conflicto". ${ }^{10}$

\section{El concepto de Patrimonio}

Con relación a la concepción de patrimonio cultural encontramos algunas propuestas:

Una concepción, propuesta por Claudio Malo en su ponencia, la que hace referencia tanto a los "elementos patrimoniales tangibles e intangibles que una colectividad elaboró a lo largo del tiempo y que conserva contenidos del pasado (...) El sentido de conservación de las elaboraciones culturales del pasado está dado por la memoria colectiva que "es el gran complemento de la temporalidad al dar unidad a nuestras existencias evitando su fragmentación y dislocación en el tiempo". Esta memoria, según Malo, tiende a ser selectiva (...) "en condiciones normales rescatamos lo que ha sido positivo y gratificante en nuestras existencias y permitimos que se evapore $y$ pierda consistencia lo insustancial y lo negativo". ${ }^{11}$

Desde esta misma visión, la Casa de la Cultura, concibe al patrimonio cultural como los sedimentos del pasado que se recrean en el presente lo que nos permite pensar el concepto de patrimonio tangible e intangible de una forma dinámica.

En la perspectiva de la Casa de la Cultura se trataría de "(...) repensar la tradición, de re-significarla en códigos contemporáneos, 
en una concepción de Patrimonio cultural como una actualización del pasado en el presente". ${ }^{12}$

Las dos versiones presentan una interesante perspectiva de trabajo y concepción cultural, pues como señala Jesús Martín Barbero: "Lo que buscamos es algo radicalmente diferente: no lo que sobrevive de otro tiempo, sino lo que en el hoy hace que ciertas matrices culturales sigan teniendo vigencia, lo que hace que una narrativa anacrónica conecte con la vida de la gente". ${ }^{13}$

Para el Banco Central, patrimonio "es el acervo valioso y sustancial que tiene una nación dentro de su herencia social".

Las versiones hacen referencia al patrimonio como producto social e histórico, sin embargo destacamos el señalado por la Casa de la Cultura pues plantea la necesidad de retomar esa gran herencia cultural, para proyectarnos en el presente y planificar nuestro futuro, como muy bien lo señala Claudio Malo:

"El patrimonio cultural sería entonces una actualización del pasado al presente $y$, en consecuencia, un referente para la planificación para el futuro". ${ }^{14}$

Pero en la práctica este enfoque de patrimonio no se evidencia en los proyectos, prevaleciendo la concepción de lo patrimonial como bienes materiales que posee la provincia. Esto se evidencia en la formulación de 8 proyectos de recuperación del patrimonio tangible (a nivel espacial y arquitectónico), 4 proyectos de recuperación de sitios arqueológicos y 2 de inventario de bienes de museos.

La preocupación por el patrimonio cultural intangible emerge, no precisamente en el sistema de "expertos", sino más bien en el planteamiento que la organización barrial realiza en torno a recuperar las tradiciones locales de los juegos populares.

Y es aquí, que el cuestionamiento a la cultura como fósil, realizado por Baudrillard es importante al evidenciar que algunas 
respetables instituciones culturales cuencanas piensan menos en el presente y futuro y más en el pasado, y de este, más en lo material que en lo intangible. Baudrillard os alertará respecto de este tema:

"Este fetichismo museístico no sólo condena a sus objetos a convertirse en residuos museológicos contemporáneos de los residuos industriales, sino que pone de manifiesto una nostalgia sospechosa. Cómo cada vez nos estamos alejando más y más de nuestra historia, estamos hambrientos de signos del pasado, en absoluto para resucitarlos, sino para llenar el espacio vacío de nuestra memoria". ${ }^{15}$

A pesar de la concepción de patrimonio cultural como una actualización del pasado en el presente, se evidencia en las prácticas culturales una suerte de regreso al pasado, no desde la necesidad de resignificarlo sino de rescatarlo. De los 25 proyectos presentados, 15 se proyectan hacia la recuperación de las tradiciones e historia, dejando de lado aspectos fundamentales del patrimonio vivo, la religiosidad, las costumbres y tradiciones locales, la organización popular, el vestido, la comida, la fiesta y los rituales, etc. que forman parte de nuestro patrimonio cultural. Casi ninguna institución presenta propuestas de investigación en torno a vitales temas del presente tales como los cambios culturales producidos por la migración internacional, el estado de las artes plásticas y las Bienales de Pintura, las pautas de consumo y la cultura local, las artesanías en el proceso de globalización, etc. Esto refuerza nuestra percepción de que la sociedad camina por un lado y las instituciones culturales por otro.

Una valoración excesiva del pasado, una nostalgia por tiempos idos, parece apropiarse de Cuenca y de sus instituciones mediadoras. Quizá la influencia de la Declaratoria puede explicarnos esta situación, pues como todos sabemos, la puesta en valor patrimonial de Cuenca se fundamentó en "su capacidad de ofrecernos un conjunto histórico, urbano, arquitectónico y paisajístico únicos". ${ }^{16}$

Lo que la UNESCO valoró es la traza urbanística colonial (materialización textual de las disposiciones fundacionales) que 
persiste hasta nuestros días. Esto, a mi modo de ver, puede marcar una concepción patrimonial que privilegia a la arquitectura y al desarrollo del espacio urbano en Cuenca obviando considerar la integralidad del patrimonio. Esto se deduce además de una primera lectura de los proyectos presentados por el Municipio de Cuenca en torno a la Declaratoria, proyectos que se inspiran en esta concepción:

"El tema del Centro Histórico de Cuenca y sus áreas especiales, asume de una manera contundente un rol prioritario en el proceso de planificación de la ciudad. (...) El Plan de Manejo del Centro histórico tiene algunos componentes. Ordenamiento territorial (fortalecer su condición de centro cultural, político, institucional y residencial (...). En relación con la edificación, promover el valor de los bienes edificados de la ciudad, programas de intervención, restauración (...) en relación a la vialidad y estacionamientos, espacio del peatón, reducción de volúmenes de circulación vehicular, regular el uso de espacios públicos para el aparcamiento (...) en relación a la vivienda, mejoramiento habitacional, rehabilitación para usos residenciales (...) equipamientos (mercados, espacios públicos, restauración urbana), transporte urbano público (descongestionamiento del centro histórico y disminución de los niveles de contaminación ambiental)". ${ }^{17}$

En la propuesta presentada ante la UNESCO hay muy pocas referencias a la cultura no material, a los bienes intangibles de la ciudad. Este fervor por lo patrimonial en sentido material, parece influir en la concepción de patrimonio a nivel local que privilegia lo monumental y por lo tanto el pasado, lo que nos permite concluir que en Cuenca hacen falta políticas de conservación "pero sabiendo que lo que hay que conservar es lo que nos queda de cultura viva, capaz no solo de dar continuidad al pasado, sino de construir el futuro". ${ }^{18}$

La tendencia -desde la Declaratoria- a valorar lo tangible es reconocida por el propio Municipio que propone por lo tanto la necesidad de rebasar esta práctica:

"Nuestra obligación como Municipio aparentemente se reduce desde el punto de vista de la Ley, a lo tangible, tendríamos que 
preocuparnos de las edificaciones, de su mantenimiento, de las calles, pero esta es una visión incompleta, por ventaja hoy hablamos de la ciudad que se ve y de la que no se ve, asimilando el concepto de ciudad a una concepción filosófica y no a una manifestación física de una realidad social. $Y$ en este espacio de lo intangible, lo que estamos propiciando es de que todos podamos hacer del título una razón para entender mejor nuestro propio origen, para entender lo que somos los cuencanos, para valorar nuestras formas de expresión cultural, para comunicar esto a nuestro propio país y fuera de él". ${ }^{19}$

Esto nos permite concluir que estamos conscientes de este acento lo que es importante pues evitará hacer de Cuenca un lugar museo en "donde se exhiben las diferencias congeladas y a donde acudimos a alimentar el recuerdo y la nostalgia". ${ }^{20}$

Tomando en consideración lo anotado, las concepciones y prácticas, el diseño de políticas en torno a lo patrimonial deberían preguntar por las dimensiones de la vida (tanto material como inmaterial) de la comunidad que necesitan ser preservadas y conservadas y por dimensiones o prácticas nuevas que deberían introducirse para dinamizar los procesos y poner a la comunidad en contacto con el mundo global con personalidad propia.

\section{IDENTIDAD:}

Conceptuar la identidad presenta diferentes ópticas y perspectivas. Por ejemplo se ha señalado que en el mundo de hoy hay varias identidades dentro de un mismo espacio cultural: identidades étnicas, de clase, de edad, de género; pero, desde otro punto de vista en cambio se plantea que por efectos de la globalización, hoy existe una desustancialización de identidades, que las identidades son móviles, son frágiles, inasibles.

Esto se complica más cuando al percibir la identidad se consideran también las estructuras económicas, políticas y sociales, las estructuras de poder pues: "Hablar de identidad cultural implica 
hablar no solo de acentos y costumbres, de músicas y artes, sino también de marginación social, de expoliación económica y de exclusión en las decisiones políticas". ${ }^{21}$

Esto evidencia la dificultad de definir la identidad en el contexto contemporáneo, pues como señala Juan Martínez: "otro elemento para comprender la identidad de finales del milenio está relacionado con la pérdida de significado tradicional de las fronteras, hoy relacionadas con el concepto de ósmosis frente al tradicional concepto del muro y la redefinición, repetitivo concepto, del sentido de la comunicación en el mundo contemporáneo". ${ }^{22}$

En efecto se asume lo que García Canclini plantea como "frontera" que ya no significa muro, separación, sino espacio de intercambio y de ósmosis cultural.

Por ello, en un primer acercamiento señalaremos que la identidad es un proceso en construcción y de-construcción permanente pues continuamente estamos recreando, perdiendo e incorporando elementos de otras culturas, activando tiempos y recursos culturales que están sedimentados 0 , construyendo nuevas señas de esa identidad.

Tanto en las propuestas presentadas como en las entrevistas en torno a identidad encontramos una tendencia general a rebasar el concepto de identidad como algo fijo, dada por la percepción antropológica de la cultura como un proceso dinámico.

"La identidad la entendemos como un conjunto de rasgos en permanente elaboración, rasgos que caracterizan a una comunidad y la diferencian de otras comunidades y sociedades". ${ }^{23}$ (Casa de la Cultura).

Estos rasgos de identidad para el Municipio se basan en que "somos producto de una simbiosis y de un mestizaje y por lo tanto hay que reconocer en la diversidad el origen de nuestra identidad cultural". ${ }^{24}$ 
Esta concepción de la ciudad como una amalgama de procesos culturales de diferentes períodos que conviven en un espacio, sus principales señas de su identidad, está presente en la propuesta de la Declaratoria, sobre todo en lo que se refiere a su patrimonio arquitectónico:

"En las 224.14 hectáreas, se encuentra su centro histórico (colonial), áreas especiales y área arqueológica" p. 29 (...) se plantea un período cañari, de gobierno inca, de conquista y colonización española". ${ }^{25}$

Estas propuestas nos permiten señalar como una primera conclusión en torno al tema de identidad, que a pesar de la influencia de la Declaratoria (identidad respecto del patrimonio tangible), la tendencia de algunas instituciones culturales es concebir la identidad de la ciudad como un proceso complejo en el que tanto la cultura material como inmaterial conforman su personalidad, pero una personalidad que no está congelada en el tiempo sino que se revitaliza, se estructura y cambia constantemente.

En el documento de análisis se advierte una segunda concepción sobre identidad que la define como "todo lo que un pueblo construye a partir de su relación con el medio físico en el cual vive, por lo que los recursos de la identidad regional serían el potencial natural de la región, el proceso histórico vivido, la constante de intercambio a nivel bioregional o interregional y los recursos ideológicos de la ciencia, la filosofía, la religiosidad y la cosmovisión andinas". ${ }^{26}$

Esta definición podría presentar el riesgo de quedarnos en una visión de cultura e identidad como algo homogéneo e internamente coherente, de concebir la región como una totalidad autónoma sin influencias externas y cambios internos.

Esta concepción de la identidad y por referencia a la territorialidad y a los procesos históricos vividos, hoy está siendo cuestionado por los procesos de re y des-territorialización, que producen por ejemplo las migraciones, los medios de comunicación, y otros fenómenos 
sociales. En el caso de Cuenca esto es evidente. Los migrantes, por ejemplo, luego de su desanclaje cultural rearman los espacios vitales del encuentro con sus semejantes y con aspectos de la cultura local en el exterior, en las plazas y parques de Madrid o Nueva York.

Desde este punto de vista parecería que hay una tendencia a buscar la identidad en referencia al pasado, a lo supuestamente puro, es decir a lo originario antes de cualquier proceso de mestizaje o hibridación cultura, o, a plantear lo identidad desde lo popular, lo vernacular pero considerado como lo indígena, lo rural, lo aborigen, lo premoderno.

"Identidad un término ambiguo, en forma simple es reconocernos quienes somos y estar orgullosos de este reconocimiento. Por tanto con esta visión de identidad nosotros promocionamos en el ámbito de la antropología y la arqueología una recuperación de nuestra herencia histórica para difundirla y sobre todo para hacer que la gente valore, se sienta orgullosa de cual ha sido su acervo cultural". ${ }^{27}$

Estas diferentes concepciones sobre identidad nos llevan a pensar en la necesidad de redimensionar la identidad desde la formación cultural de Cuenca y del Azuay por lo que sería importante analizarla a la luz de la propuesta de De Williams y sus propuestas de análisis la topología de las formaciones culturales en las que se podría reconocer estratos: arcaico, residual, emergente:

"Arcaico: lo que sobrevive del pasado en cuanto pasado, objeto únicamente de estudio de rememoración; residual: lo que formado efectivamente en el pasado se halla hoy dentro del proceso cultural (...) como efectivo elemento del presente (...) y emergente, que es lo nuevo, el proceso de innovación en las prácticas y los significados". ${ }^{28}$

Este sería un interesante tema de análisis de la cultura local. Por ahora y en un primer acercamiento al tema considero que en los proyectos presentados por las instituciones culturales cuencanas la identidad por referencia al pasado es la que predomina, y de este pasado se trataría de rescatar el patrimonio tangible que se enmarca 
más en el estrato arcaico (5) y residual (9), que en el emergente. Esta tendencia a definir la identidad con respecto al pasado, a replegarse en él, podría tener su explicación en la valoración por parte de la UNESCO o en los procesos de recuperación y resignificación del territorio como espacio vital desde el punto de vista político y cultural que probablemente genera la globalización.

Frente a la tentación de asumir la identidad por referencia al pasado, Juan Martínez nos advierte sobre la necesidad de pensar en los procesos de construcción de identidad futura, advertencia con la que estamos de acuerdo:

"El futuro de la identidad local dependerá de nuestra capacidad de asumir una posición definida en el contexto de un mundo interconectado. Es decir que Cuenca (...) sociedad compleja, parte moderna, parte arcaica, parte desesperadamente contemporánea, solamente mantendrá su identidad local en el contraste con nuestra capacidad de asumir lo cosmopolita". ${ }^{29}$

\section{Cultura y Desarrollo}

Es evidente que todavía estamos muy lejos de generar una vinculación entre cultura y desarrollo local-global.

En el documento analizado solo una de las ponencias pone énfasis en la vinculación entre los conceptos de identidad, patrimonio y desarrollo, pues los tres conceptos actúan entre sí. En cambio en el análisis de los proyectos solo uno (referido a turismo) hace referencia al desarrollo local.

Dominique Gomis plantea que hay dos "ideas de desarrollo: la una, vinculada con el modelo globalizante de tipo occidental y que, en los países pobres, actúa desde lo exterior. La otra, relativa a las distintas vías tomadas por los pueblos, que corresponde a la respuesta que dieron estos, a la enorme biodiversidad del planeta, a través de ciencias y tecnologías aplicadas y apropiadas a cada caso. Este 
desarrollo parte de lo interior". ${ }^{30}$

Desde esta perspectiva las huellas de un pasado regional común -ya presente en otras apreciaciones locales- con su fuerte componente de cosmovisión andina podrían potenciar un nuevo desarrollo.

Con estos antecedentes, la autora citada se pregunta: “ $¿$ hacia qué modelo de desarrollo autónomo ir: desde lo exterior, en donde la regionalización sigue causando en la práctica, problemas de estructuración y conceptualización. O bien desde lo interior, a partir de las experiencias vividas por un substrato cañari, enriquecido por el mestizaje y ciertos elementos occidentales modernos, y en donde se hallarían las ventajas y se convertirán las desventajas en oportunidades de equidad?". ${ }^{31}$

Si bien en este discurso se ubica una concepción de desarrollo y cultura como dos caras de una misma moneda, se considera en cambio que la mundialización o globalización no nos ha tocado, que permanece fuera en el tiempo y en el espacio, como algo externo, como una amenaza a la cual hay que responder desde la identidad cultural local.

Esta visión sobre nuestra realidad cultural sería importante debatirla pues, a mi modo de ver, no da cuenta de los cambios producidos a lo largo de nuestra historia, y no considera que lo global y lo local está seriamente articulados aquí y ahora, que lo "externo" que pretende homogenizar está produciendo "mundos internos", respuestas locales activas, metabolización cultural pues no hay recepción pasiva y que hay múltiples influencias, mecanismos y canales de paso de lo global a lo local y viceversa.

"Es un error pensar que la globalización sólo concierne a los grandes sistemas, como el orden financiero mundial. La globalización no tiene que ver sólo con lo que hay "ahí fuera", remoto y alejado del individuo. Es también un fenómeno de "aquí dentro", que influye en los aspectos íntimos y personales de nuestras vidas". ${ }^{32}$ 
El tema de cultura y desarrollo es así un tema complejo que requiere de acercamientos diversos y multidisciplinarios, significa un nuevo objeto de investigación que implica considerar una serie de factores y fenómenos tales como homogeneización y la heterogeneidad, las circulaciones interculturales, las relaciones de poder, las perspectivas de desarrollo, la vinculación entre el nivel micro y macro social, entre otros.

Sin embargo tanto en las propuestas del programa como en las entrevistas no hay propuestas sólidas.

En la entrevista, el Representante del Municipio de Cuenca hace referencia tan solo al hecho de que lo global en referencia a lo local vehicula procesos de intercomunicación.

"La globalización tiene quizá como único mérito facilitar las comunicaciones con medios importantes como el internet y otros equivalentes y facilitar el intercambio de valores locales que empiezan a acercar mucho más a los ciudadanos. Esta proximidad de gobiernos locales va a tener importantísimas connotaciones en humanizara la globalización". ${ }^{33}$

EIBanco Central tiene igual percepción y acentúa en la necesidad de apropiación tecnológica en lo local para tener un impacto en lo global:

"Estamos incursionando en todo lo que es la tecnología informativa y telecomunicaciones para tener un impacto en lo local y a su vez en lo local para tener un impacto en lo global". (Banco Central).

Pero es evidente que el tema empieza a ser asumido como importante, por lo que el Municipio de Cuenca señala la necesidad de "armar sostenidamente un plan de difusión cultural" para la ciudad, lo que coincide con el planteamiento de otras instituciones culturales cuencanas.

La vinculación entre cultura y desarrollo local es un tema vital 
para posicionarnos en el contexto de un mundo globalizado desde una mejor perspectiva, pues como lo señala Jesús Martín Barbero, "la región está significando un lugar clave a la hora de pensar la resistencia y la creatividad frente a la homogeneización". ${ }^{34}$

La importancia de lo cultural en el desarrollo de los pueblos ha implicado un largo proceso en el que la dimensión cultural poco a poco ha sido asumida como una dimensión sustantiva y no como un mero elemento adicional a los indicadores básicamente económicos. Pues la diferencia cultural, lo diverso, "lo otro", como valor agregado puede generar un impacto importante en las economías locales y por ende beneficiar a todos los actores locales.

Pero no basta con esta constatación. Para que el capital simbólico que poseemos se potencie se requiere organización y planificación, en primer lugar para volverlo apto para el mercado mundial, espacio de mucha competitividad en este campo, y en segundo lugar, propiciar estrategias adecuadas para un crecimiento equilibrado que considere todos los factores: el patrimonio natural y cultural, la población, los turistas, como un todo.

Ahora bien, la propuesta desarrollo y cultura podrían analizarse bajo dos ópticas: como una oportunidad y como una amenaza. Oportunidad en el sentido de que lo cultural puede actuar como un elemento que active las potencialidades locales, genere procesos de autoestima, movilice socialmente, procure nuevas formas de ejercicio de la democracia y construcción de la ciudadanía. La amenaza en cambio tiene que ver con el gran mercado globalizado en el que la especificidad cultural puede perder sus verdaderos sentidos y transformarse en una mercancía más, en un fetiche, comercializado por las transnacionales.

Para evitar esta última posibilidad es urgente retomar propuestas que replantean el desarrollo desde nuevas perspectivas, superando una racionalidad económica que se sustenta en la acumulación indiscriminada, en el mejoramiento de los indicadores macroeconómicos y en la eficiencia al margen de las necesidades 
humanas por otra racionalidad que se orienta al mejoramiento de la calidad de vida de la población, el fortalecimiento de los espacios locales y la multiculturalidad, entre otros importantes aspectos.

"La referencia a la calidad de vida como concepto y a la vez como objetivo, nos aproxima a la existencia de un muy amplio y diverso conjunto de necesidades, que al ser abordadas integralmente, ubican en similares niveles de prioridad, importancia y emergencia, tanto las necesidades materiales como las necesidades espirituales de la población". ${ }^{35}$

Se trataría por lo tanto de generar una nueva visión del desarrollo local, con nuevas concepciones en torno a las necesidades y a los satisfactores, en la que el ámbito cultural es un factor de primer orden para potenciar esa integralidad.

Para esto se requiere que temas como cultura, identidad, patrimonio y desarrollo tengan una coherencia conceptual pero además se requiere de un proceso de construcción colectiva en el que la conceptualización, la investigación sobre lo que está ocurriendo a nivel local en el ámbito cultural, la construcción de políticas culturales, la organización y la gestión son partes de un proceso clave.

\section{CONCLUSIONES}

- En elámbito conceptual, en las instituciones culturales cuencanas, se ha generado un cambio en torno a la concepción de cultura, pasando desde una concepción tradicional ligada a las bellas artes a una concepción antropológica que sirve de referencia para el trabajo y prácticas institucionales. Sin embargo, dada la complejidad de las sociedades actuales, pensamos que es importante abordar lo cultural desde un punto de vista más integral, por lo que consideramos que la sociología de la cultura puede significar un importante aporte en este campo. 
- Se ha desarrollado una concepción sobre patrimonio que alude tanto a elementos tangibles como intangibles de la cultura cuencana. Sin embargo en la práctica, quizás por influencia de la Declaratoria, las instituciones siguen generando sus propuestas en torno al rescate del patrimonio material, dejando de lado el patrimonio inmaterial, tan rico y diverso en nuestra provincia y región.

- La concepción de patrimonio cultural como actualización del pasado en el presente no logra desarrollarse a través de proyectos. Todos ellos se dirigen más bien a la recuperación de tradiciones históricas, especialmente del pasado colonial, dejando de lado el patrimonio vivo.

- Asimismo, no hay ni un solo proyecto de investigación sobre lo que está sucediendo a nivel de la cultura local ni propuestas ni proyecciones futuras, lo que es preocupante dadas las condiciones de frontera que se han desarrollado en Cuenca por efectos de la migración, o la propia Declaratoria que podría motivarnos a desarrollos culturales diferentes.

- A nivel teórico se conceptúa la identidad como un proceso permanente de construcción y deconstrucción y por ella la necesidad de pensar en el futuro de la identidad local en el contexto de un mundo interconectado y globalizado. Pero la tendencia de vivir la identidad en referencia al pasado es evidente. En este tema sería muy importante realizar investigaciones más profundas sobre cómo la globalización presiona a las culturas locales a un repliegue sobre sí mismas y básicamente por referencia al pasado.

- La necesidad de vincular desarrollo local y cultura es urgente. Sin embargo las instituciones mediadoras no han logrado advertir esta necesidad y no enmarcan su gestión en este campo. Esto no solamente como uno de los componentes del desarrollo local o como un tema adscrito al mismo sino considerando lo cultural como una clave de resistencia y creatividad frente a la 
mundialización, con el propósito de revertir los efectos desocializadores del neoliberalismo.

- Se impone por lo tanto la necesidad de generar un proyecto de coordinación y de formulación de un Plan de Desarrollo para la cultura local, que sea capaz de construir efectivamente una nueva ciudad a partir de lo simbólico, que esté enmarcado en una concepción de desarrollo alternativo y que pueda propiciar el desarrollo de políticas culturales con la participación propositiva de los diferentes sectores sociales.

\section{NOTAS}

1. Giddens, Anthony: "Consecuencias de la Modernidad". Alianza Editorial, 1990, pág. 689.

2. Escobar Ticio, "El Mito del Arte y el Mito del Pueblo". Ediciones Del Sol, Buenos Aires, 1991, pág. 87.

3. Habit-Com, UNESCO, PIDC Directorio de Instituciones y Proyectos de Desarrollo Cultural, Quito, 1997.

4. R. Williams R., citado por Ariño, Antonio, en "Sociología de la cultura, la constitución simbólica de la sociedad", Editorial Ariel S.A., Barcelona 1997, pág. 19.

5. Malo G., Claudio. "Patrimonio Cultural Material e Intangible" ponencia presentada en el Programa Interinstitucional "La Identidad en el Patrimonio, Cuenca, junio 2000, pág. 2.

6. Jaramillo, Diego, Presidente de la asa de la Cultura, Entrevista, Mayo $17 / 2001$.

7. Cordero, Fernando, Alcalde de Cuenca, Entrevista, Mayo 18/2001.

8. Cordero, Ibid.

9. Abad, Andrés, Director de Cultura del Banco Central, Entrevista, Mayo $17 / 2001$. 
10. Thompson, citado por Ariño, Antonio: Sociología de la Cultura, Barcelona, Editorial Ariel S.A., 1997, pág. 36.

11. Malo, Claudio: Ibid, pág. 3.

12. Programa Interinstitucional "La Identidad en el Patrimonio", Cuenca, junio 2000, pág. 16.

13. Martín-Barbero, Jesús: "De los medios a las mediaciones", Convenio Andrés Bello, Bogotá, s.f., pág. 30.

14. Malo, Claudio: Patrimonio cultural intangible y globalización, pág. 37.

15. Baudrillard, Jean, "La llusión del Fin", Anagrama S. A., Barcelona, 1993, pág. 115.

16. Municipalidad de Cuenca, "Propuesta de inscripción del Centro Histórico de Cuenca- Ecuador en la lista de patrimonio mundial, pág. 11.

17. Ibid, Plan, págs. 48 a 50.

18. Martín-Barbero, Jesús, PRE - Textos, Universidad del Valle, Cali, 1996, pág. 138.

19. Cordero, entrevista, ibid.

20. Baudrillard, lbid, pág. 80.

21. Martín-Barbero, lbid, pág. 89.

22. Martínez Borrero, Juan, "Hacia la deconstrucción de la identidad local", ponencia incluida en el Programa Interinstitucional "La Identidad en el Patrimonio", Cuenca, junio 1999, pág. 13.

23. Jaramillo, entrevista, ibid.

24. Cordero, entrevista, ibid.

25. Propuesta de inscripción del Centro Histórico de Cuenca-Ecuador en la lista de patrimonio mundial. Municipalidad de Cuenca, pág. 39.

26. Gomis, Dominique, "Identidad, Patrimonio y Desarrollo”, Cuenca, junio 1999, pág. 7.

27. Abad, entrevista, ibid.

28. De Williams, citado por Martín-Barbero, Jesús, Ibid, pág. 107.

29. Martínez, lbid, pág. 15.

30. Gomis, Dominique, lbid, pág. 7.

31. Ibid, pág. 8.

32. Giddens, Anthony, "Un mundo desbocado. Los efectos de la Globalización en nuestras vidas", Tahúras, Madrid, 1999.

33. Cordero, entrevista, ibid.

34. Martín-Barbero, ibid, pág. 87.

35. Astudillo Romero, Jaime, Plan de Desarrollo Cultural para Cuenca y la región. Universidad de Cuenca, 1995, pág. 13. 


\section{BIBLIOGRAFÍA}

Astudillo Romero, Jaime. Plan de Desarrollo Cultural para Cuenca y la región, Universidad de Cuenca, Cuenca, 1995.

Baudrillard, Jean, "La llusión del Fin”, Anagrama S.A., Barcelona, 1993.

Escobar, Ticio, "El Mito del Arte y el Mito del Pueblo", Ediciones Del Sol, Buenos Aires, 1991.

Giddens, Anthony, "Consecuencias de la Modernidad", Alianza Editorial, 1990.

Gomis, Dominique, "Identidad, Patrimonio y Desarrollo". Ponencia presentada en el Programa Interinstitucional "La Identidad en el Patrimonio", Cuenca, junio 1999.

Habit-Com. UNESCO, PIDC Directorio de Instituciones y Proyectos de Desarrollo Cultural, Quito, 1997.

Malo González, Claudio, "Patrimonio Cultural Material e Intangible", ponencia presentada en el Programa Interinstitucional "La Identidad en el Patrimonio", Cuenca, junio, 2000.

Malo González, Claudio. "Patrimonio cultural intangible y globalización".

Martín-Barbero, Jesús, "De los medios a las mediaciones”, Convenio Andrés Bello, Bogotá, s.f.

Martín-Barbero, Jesús, PRE-Textos, Universidad del Valle, Cali, 1996.

Martínez Borrero, Juan, "Hacia la deconstrucción de la identidad local”, ponencia incluida en el Programa Interinstitucional "La Identidad en el Patrimonio", Cuenca, junio 1999.

Municipalidad de Cuenca, "Propuesta de inscripción del Centro Histórico de Cuenca-Ecuador en la lista de patrimonio mundial". 